\title{
Detection of putative stem cell markers, CD44/CD133, in primary and lymph node metastases in head and neck squamous cell carcinomas. A preliminary immunohistochemical and in vitro study
}

\author{
Mannelli, G., ${ }^{*}$ Magnelli, L., ${ }^{\dagger}$ Deganello, A., * Busoni, M., Meccariello, G., * Parrinello, G.* \& Gallo, O.* \\ ${ }^{*}$ First Clinic of Otorhinolaryngology Head and Neck Surgery, University of Florence, Azienda Ospedaliera Universitaria Careggi, \\ ${ }^{\dagger}$ Department of Sperimental Pathology and Oncology Medical School, University of Florence, Florence, Italy
}

Accepted for publication 31 December 2014

Clin. Otolaryngol. 2015, 40, 312-320

Objectives: Investigators hypothesized that cancer stem cells (CSCs) could play a role in determining cancer progression by metastasizing to cervical lymph node $(\mathrm{N}+)$ and then influencing prognosis of head and neck squamous cell carcinomas (HNSCCs) patients.

Design: To identify CSCs in HNSCCs and their clonogenic capacity.

Setting: In vitro study.

Participants: Putative CSCs from 29 primary HNSCCs and 19 corresponding node metastases were analyzed.

Main outcome measures: Immunohistochemical (IHC) was performed, and CSCs' clonogenic in vitro capacity was tested; ones epithelial nature of cancer cells forming colonies was confirmed by a second IHC, fluorescence-activated cell sorting (FACS) analysis helped in counting CD44/CD133-
CSCs markers percentage expression in HNSCC tumourderived cultures.

Results: Immunohistochemical showed CD44 (93.1\%) and CD133 (10.34\%) expression; FACS-analysis showed the enrichment of CD44/CD133 cancer cells, with the highest clonogenic capacity of CD44+-subpopulation; a higher CD44 rates were documented from $\mathrm{N}+$ subcultures than from original tumours $(P<0.05)$.

Conclusions: A putative cancer stem-like cell population is detectable in HNSCCs, and our findings show their in vitro clonogenic capacity by demonstrating that $\mathrm{CD} 44+$-cultured cells are the main population proliferating obtained by $\mathrm{N}+$ HNSCC metastases, emphasizing their possible role in tumour progression.
Head and neck squamous cell carcinoma accounts approximately 500000 new cases per year. ${ }^{1}$ Despite advances in treatment, survival rates have not improved significantly in more than 30 years; mortality is mainly related to metastases' development, therapy resistance, local and regional recurrences. Clinical and pathological prognostic factors such as tumour stage, lymph node involvement, postsurgical positive margins and histological grade, lack sensitivity and accuracy in the clinical setting and, with the exception of disease stage, are infrequently used to guide treatment decisions. $^{2-4}$ Better understanding of biologic behaviour of Head and neck squamous cell carcinoma is required to define novel therapeutic strategies.

Over the last two decades, advances in tumour biology have led to identify in many cancers, including Head and neck squamous cell carcinomas, a subpopulation of cancer cells with stem properties, which is able to undergo

Correspondence: G. Mannelli, First Clinic of Otolaryngology Head-Neck Surgery, University of Florence, Azienda Ospedaliera Universitaria Careggi, Via Largo Brambilla 3, 50134 Florence, Italy. Tel: (0039) 0557947988; Fax: (0039) 055435649; e-mail: mannelli.giuditta@gmail.com self-renewal and differentiation, to initiate tumourigenesis and hence to support tumour growth, progression and chemo-/radioresistance. ${ }^{5,6}$ This cancerogenesis hypothesis considers that stem cells or cells that acquired the selfrenewal ability tend to accumulate genetic alterations over long periods of time, evading the strict control of their microenvironment and giving rise to tumoural evolution. ${ }^{7}$ Numerous hypothesis exists about the origin of cancer stem cells, ${ }^{8}$ and the difficulty in studying normal stem cells and cancer stem cells has led to use empirical models of isolation of cancer stem-like cells by specific cell-surface markers and the characterisation of their behaviour by in vitro cultures and in vivo self-renewal assays. CD44 and CD133 are the main cell-surface markers associated with stem cell characteristics. $^{9-12}$ CD133 or prominin 1 (PROM1) was discovered as a marker of normal hematopoietic stem cells, and its power to identify cancer stem-like cells has been confirmed recently. ${ }^{13}$ CD133's display has been found in several solid tumours of different anatomical sites, head and neck district included. ${ }^{14-18}$ CD44, receptor of hyaluronic acid, seems to be involved in cell adhesion, migration and metastasis of cancer cells. ${ }^{19}$ Various CD44 variant isoforms are differentially 
expressed in normal and malignant cells. Overexpression of several CD44 variant isoforms has been associated with tumour progression, suggesting that these isoforms may have unique signalling properties. Several human tumours show CD44 altered expression and translation, with CD44 changing during tumour progression. ${ }^{20-22}$ All of these investigations suggest that different stem cell markers could be expressed in various tumours by the cancer stem cells, but their significance remains to be determinated. Therefore, it is possible that each solid tumour could show a specific cancer stem cell phenotype. ${ }^{23}$

The main published articles show the presence of a diffuse positivity of CD44 antigen (>95\%) in Head and neck squamous cell carcinomas, which identify cancer cells with clonogenic in vitro properties, while few CD44 and CD133 co-expressing cells are encountered $(<10 \%){ }^{24}$

The role of cancer stem cells in metastasis is not clear yet, but it is likely they are the cells responsible for their development. Cancer stem cells would migrate and attach a new location, and local conditions should able to stimulate and support cancer stem cells and the production of their progenitors. ${ }^{25-27}$ Then, an evaluation of the cancer stem cells' role in metastasis will produce new insights into this process and will lead to new treatments in order to prevent or eliminate metastatic disease.

It is well known that cancer stem cells and normal stem cells share some properties, and on the basis of these remarks, our aim was, first of all, to investigate the potential role of cancer stem cells in tumour progression, by detecting the presence of phenotype stem-like characteristics, by CD44 and/or CD133 antigens expression, in cancer specimens from Head and neck squamous cell carcinomas. Moreover, the parallels existing between normal somatic stem cells and cancer stem cells suggest that the principles of normal stem cell biology may be usefully applied to studies of cancer stem cell identification and their roles in tumour development and progression, ${ }^{28}$ and current methods for determining whether cells isolated from solid tumour are cancer stem cells consist of purification of these one based on such stem properties as their ability to form spheres in culture. ${ }^{12,29,30}$ To better address this issue, we harvested from both primary and lymph node metastases $(\mathrm{N}+)$ tumour surgical specimens, cancer cell cultures to comparatively evaluate their phenotype and clonogenic in vitro potential.

\section{Materials and methods}

The study was conducted on tumour specimens, obtained in sterile conditions at the operating table, during the tumour surgical excision from 29 consecutive patients affected by primary and not previously treated Head and neck squamous cell carcinoma, from April 2008 to July 2009 at our institution. Patients previously treated by surgery, radiotherapy and/or chemotherapy, tumour recurrence or persistence, second primary tumour, synchronous or metachronous tumours were all considered as exclusion criteria.

The study design included (i) immunohistochemical analysis on sections from primary Head and neck squamous cell carcinoma and their lymph node metastasis to provide the presence of cancer cells with stem-like phenotype characteristics (CD44+ CD133+) and the evidence for a developmental hierarchy in Head and neck squamous cell carcinoma, such as in normal epithelial tissue; (ii) ones identified stem-like cancer cells, the second step was to perform in vitro cultures of spheres cells by testing the cancer stem-like clonogenic capacity; (iii) a second immunohistochemical study on cell cultures was used to exclude a possible component of flogist cells and to confirm the epithelial nature of cells forming colonies; (iv) fluorescence-activated cell sorting analysis was used to count the CD44+ CD133+ cancer cells percentage forming cell colonies.

\section{Ethical considerations}

This study was approved by the Florence Universitarian Hospital IRB, and all participants signed an informed consent agreement. All patients gave their consent to undergo the procedure and to treat their personal details. No more to declare.

\section{Immunohistochemistry}

Frozen sections from neoplastic fragments were embedded in cold acetone for $1 \mathrm{~min}$ and dried for $10 \mathrm{~min}$ at $37^{\circ}$. The sections were rinsed in distilled water for $5 \mathrm{~min}$ and then in phosphate buffer ( $\mathrm{pH} 7.4$ ); immunohistochemical studies were performed on formalin-fixed, paraffin-embedded tissue sections ( $5 \mu \mathrm{m}$ of thickness) using the technique of streptavidin-biotin-peroxidase (Labvision Co, Freemont, CA, USA).

Immunohistochemistry-CD133. CD133 primary antibody (Miltenyi Biotech, Bologna, Italy) was used at a dilution of $1: 100$ with incubation for $1 \mathrm{~h}$ in environmental temperature. Further, these sections were counterstained. Sections without primary antibodies served as negative controls.

Immunohistochemistry-CD44. Specimens were deparaffinazed with xylene, rehydrated in serial graded waterethanol solution and then rinsed in 3\% hydrogen peroxide solution to eliminate endogenous peroxidase. The sections were then heated in a microwave oven for $35 \mathrm{~min}$ at $300 \mathrm{~W}$ in citrate buffer $(0.1 \mathrm{Mol}, \mathrm{pH} 6.0)$, placed in a phosphate- 
buffered saline ( $\mathrm{pH} 7.6)$, and then CD44, CD44v3 and CD44v6 monoclonal primary antibodies were applied ( $\mathrm{R} \&$ D Systems, Abingdon, UK) at a dilution $1: 1000$ at $4^{\circ}$ overnight. Sections were counterstained. Sections without primary antibodies served as negative controls.

\section{Cell cultures}

We grouped the establishment of cell cultures to characterise the cancer cells' biology and their potential stem properties. Biopsy-proven squamous cell carcinoma nature of the primary tumour or of the corresponding lymph node metastasis where it was present was surgically resected from each patient and immersed into sterile phosphate-buffered saline ( $\mathrm{pH} 7.4)$ supplemented with pen-strep $(100 \mathrm{U} / \mathrm{mL}$ pen $100 \mu \mathrm{g} / \mathrm{mL}$ strep, Euroclone) and amphotericin $(250 \mu \mathrm{g} / \mathrm{mL}$, Euroclone $)$ and sent to the department of Experimental Pathology and Oncology. Specimens were cut into small pieces $(1 \mathrm{~mm})$ and shaked for $4 \mathrm{~h}$ at $37^{\circ} \mathrm{C}$ in phosphate-buffered saline supplemented with $1 \mathrm{mg} / \mathrm{mL}$ collagenase III (Biochrome AG), to perform enzymatic digestion. Each digestion was divided into two aliquotes that were centrifuged at $1100 \mathrm{rpm}$ : revolutions per minute (10 $\mathrm{min}$ ), to remove the digestion buffer. One wad was resuspended in freezing medium ( $90 \%$ FCS, $10 \%$ DMSO) and stored under liquid nitrogen. This sample was used as 'time 0' (early culture), as it is representative of biopsy whole cell population. Cells from the second wad were resuspended in culture medium (DMEM supplemented with $20 \%$ FCS and pen-strep and amphotericine, as above), seeded in a Petri dish and incubated at $37^{\circ} \mathrm{C}$ in a $5 \% \mathrm{CO} 2$ humidified atmosphere. After a few days, adherent cells were rinsed with phosphatebuffered saline and fresh medium wad added. When subconfluent, cells were harvested by trypsin-EDTA treatment $(0.5: 0.2 \mathrm{mg} / \mathrm{mL})$, resuspended in freezing medium and stored under liquid nitrogen. For typisation, an aliquote of cells were seeded on a glass slide. When subconfluent, cells were fixed for 10 min with ice-cold 90\% ethanol for immunohistochemistry. Major difficulties in setting up $\mathrm{H} \& \mathrm{~N}$ cancer cell lines from biopsies were sterility failure and fibroblast contamination. Sterility was achieved in about $40 \%$ of cultures. $^{9-12}$ After 3 weeks of culturing, we obtained 'late culture'.

\section{Immunohistochemistry on cell cultures}

Immunohistochemistry of early and late cell cultures used the same antibodies against CD44 and CD133.

Immunohistochemistry-CD133. CD133 primary antibody (Miltenyi Biotech) was used at a dilution of $1: 100$ with incubation for $1 \mathrm{~h}$ in environmental temperature.
Immunohistochemistry-CD44. CD44, CD44v3 and CD44v6 monoclonal primary antibodies were applied $(\mathrm{R} \& \mathrm{D}$ Systems) at a dilution $1: 1000$ at $4^{\circ}$ overnight.

Moreover, we verified the epithelial nature of cell cultures, by detecting the presence of cytoplasmic epithelial cytokeratin.

\section{Fluorescence-activated cell sorting analysis}

Each obtained Head and neck squamous cell carcinoma cultures were washed with phosphate-buffered saline and fixed with phosphate-buffered saline supplemented with $3 \%$ paraformaldehyde $\left(10 \mathrm{~min}, 4^{\circ} \mathrm{C}\right)$ and incubated (i) none, (ii) anti-CD133-PE (clone-AC133; Miltenyi Biotec), (iii) anti-CD44-APC (clone-IM7; eBioscience, San Diego, CA, USA) and (iv) both anti-CD133-PE and anti-CD44-APC. Cell staining was in accordance with manufacturer's instructions. Cytofluorimetric analysis was carried out with a FACSCanto apparatus (Becton Dickinson, La Jolla, CA, USA) Coherent Sapphire Solid State laser $(\lambda=488 \mathrm{~nm})$ and a JDS Uniphase HeNe laser $(\lambda=633 \mathrm{~nm})$, computed by the BD Diva 6.1.2. software (Becton Dickinson). Sections without primary antibodies served as negative controls.

\section{Statistical analysis}

Results were evaluated using analysis of variance, chi-square and Student $\mathrm{t}$ technique for paired data. Statistical significance was set for $P$-values $P<0.05{ }^{31}$

\section{Results}

Among 29 Head and neck squamous cell carcinoma patients, 19 were male and 10 female, with a mean age of 63.08 years $(60.06 \pm 14.05 \mathrm{SD}$ years for male and $66.10 \pm 10.88$ SD for female). All of these patients were affected by newly diagnosed Head and neck squamous cell carcinoma. Head and neck squamous cell carcinomas originated from the oral cavity in 12 (two of these were from the alveolar process, two from the buccal mucosa, four from retromolar trigone, four oral tongue), in six cases from oropharynx (three of these were from the tonsil, one of the soft palate, one from tongue base and one from the vallecula), seven were laryngeal tumours, and in four cases, we studied the cervical lymph node metastases from primary unknown. Clinical data were collected, including age, sex, alcohol and tobacco consumption (cut-off $=$ almost 10 pack/years), cancer family history, comorbidities (liver pathology, cardiovascular pathology, glaucoma, mellitus diabetes); TNM stages and the primary tumour sites are shown in Table 1. 
Table 1. Clinical parameters, TNM stage and primary tumour sites.

\begin{tabular}{ll} 
Clinical parameters & Number of patients: $\%)$ \\
\hline Sex & \\
M & $19(65.5)$ \\
F & $10(34.5)$ \\
Age & \\
M & $60.06 \pm 14.05 \mathrm{SD}$ \\
F & $66.10 \pm 10.88 \mathrm{SD}$ \\
Alcohol consumption & $11(38)$ \\
Yes & $18(62)$ \\
No & \\
Tobacco consumption & $21(72.4)$ \\
Yes, p/y = 25.62 \pm 29.93 SD & $8(27.6)$ \\
No & \\
Alcohol + tobacco consumption & $11(38)$ \\
Yes & $18(62)$ \\
No & \\
Cancer family history & $15(51.7)$ \\
Yes & $14(48.3)$ \\
No & $13(44.8)$ \\
Comorbidities & $16(55.2)$ \\
Yes & $29(100)$ \\
No & \\
& \\
\hline
\end{tabular}

\begin{tabular}{llllllll}
\hline TNM & \multicolumn{1}{l}{ T2 } & T3 & T4a & T4b & Tx & Total \\
& T1 & T2 & & & & & \\
N0 & 3 & 4 & 2 & & & & 9 \\
N1 & 4 & 6 & & 1 & & 1 & 12 \\
N2a & & 2 & 1 & & & 1 & 4 \\
N2b & & 1 & & & 1 & 1 & 3 \\
N2c & & 1 & & & & & 1 \\
N3 & & & & & & & \\
Total & 7 & 14 & 3 & 1 & 1 & 3 & 29 \\
\hline
\end{tabular}

\section{Primary sites}

Number of patients

\begin{tabular}{ll} 
Oral cavity & 12 \\
Alveolar process & 2 \\
Oral mucosa & 2 \\
Retromolar trigone & 4 \\
Oral tongue & 4 \\
Oropharynx & 6 \\
Tonsil & 3 \\
Soft palate & 1 \\
Tongue base & 1 \\
Vallecula & 1 \\
Larynx & 7 \\
Supraglottic & 3 \\
Glottic & 2 \\
Subglottic & 2 \\
TxN+ & 4 \\
\hline
\end{tabular}

\section{Immunohistochemistry}

CD133. Immunohistochemical study showed positivity for CD133 in only three of the 29 cases analysed (10.34\%). The percentage of positive tumour cells was variable (from five to $30 \%$ of counted cells), indicating that only a fraction of the total tumour cells expressed this marker. In our series, we found the highest CD133 expression in tumour specimens derived from cervical lymph node metastases (10.5\%, two of $19 \mathrm{~N}+$ ), while the CD133 primary tumour specimens expression found was of $3.4 \%$ (one case of 29 primary tumour specimens $)(P<0.05)$.

CD44s. It was overexpressed in 27 of the 29 cases examined (93.1\%). In particular, all three tumours CD133 positive were CD44 positive too. Unlike CD133, immunohistochemical showed high rate of CD44-positive cancer cells, either from biopsy of primary tumour or from metastases.

Moreover, we obtained a strong CD44-positive basal cell layers in tumours moderately or highly differentiated, while in the more keratinised and differentiated cells, this marker was absent (Fig. 1).

The immunohistochemical analysis of consecutive tumour specimen slices (Fig. 2) suggests a potential coexpression of CD44/CD133 antigens.

\section{Cell cultures}

The primary Head and neck squamous cell carcinoma cells isolated from six patients (Table 2) could be passed for more than 16 passages without loss of growth, viability or the morphological features of the parental cells. Free-floating spherical colonies were obtained from tumour biopsies taken from primary tumours in four of the six cases (T1N1,

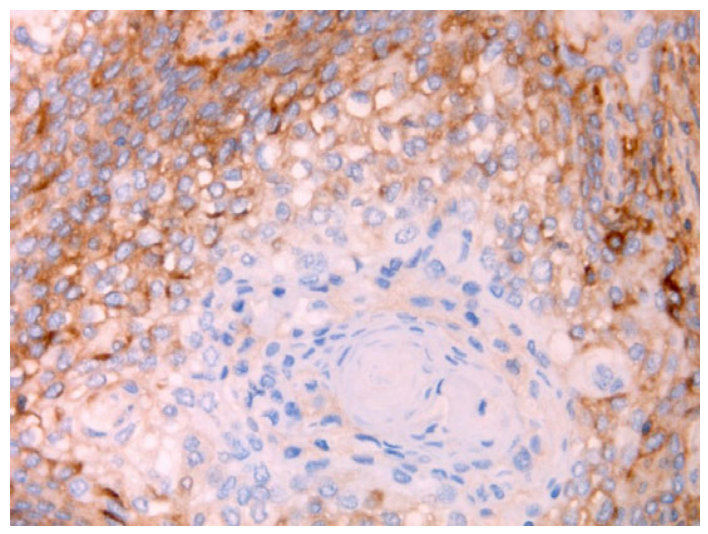

Fig. 1. Strong CD44-positive basal cell layer suggesting a clear topographical cancer stem cells redistribution. While this marker was absent in the more keratinised and differentiated cells. 

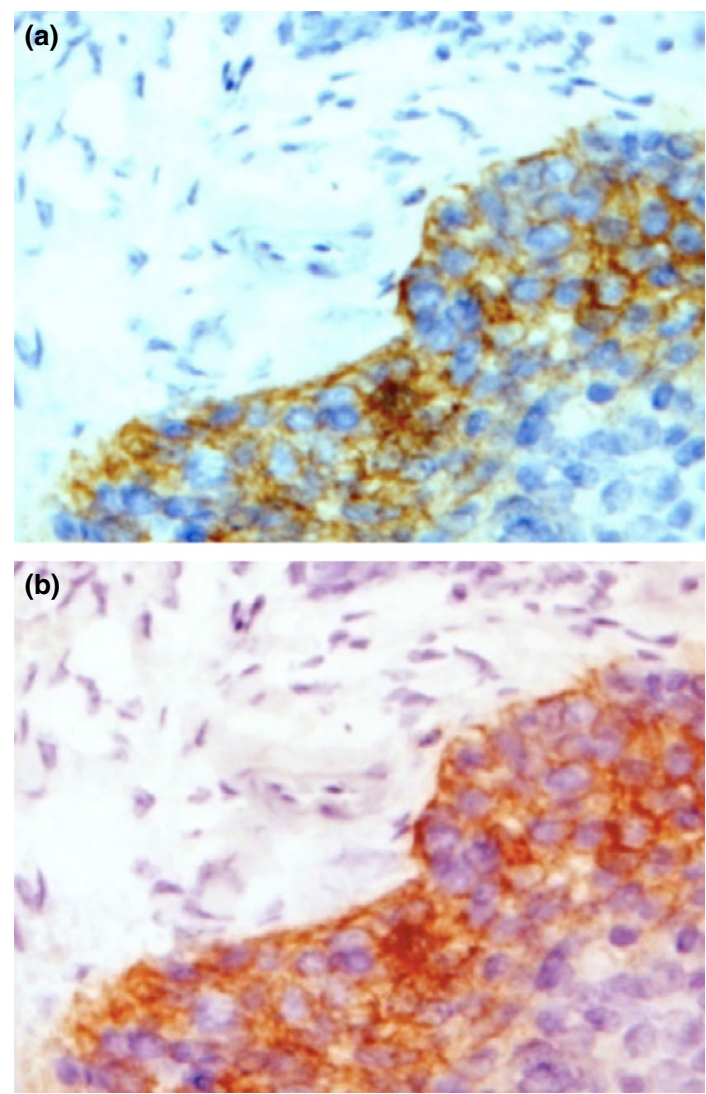

Fig. 2. CD44 and CD133 co-expression.

T2N0, T4bN2b, T4aN1) and from the lymph node metastases in two of the six cases (TxN1M0 and T2N2b). Instead of getting a total of 12 cultures, including six early and other six late cultures, the final number was of only eight cultures overall, of which six were early cultures and only two were late cultures, because of bacterial contaminations and that the remaining subcultures did not reach the optimal period of incubation that they can refer to various studies planned.

\section{Immunocytochemistry on cell cultures}

We verified the epithelial nature of cell cultures, by detecting the presence of cytoplasmic epithelial cytokeratin, to exclude the successful replication of the cellular inflammatory component and/or fibroblasts (Fig. 3). Then, the immunohistochemical analysis on cell cultures searching for both markers' expression, CD44 and CD133, confirmed the highest expression of CD44 in these cancer cell culture populations, analogously to immunohistochemical on fresh tumour specimens.

\section{Fluorescence-activated cell sorting analysis}

Fluorescence-activated cell sorting analysis was performed on six early cultures (Table 2), and on their late cultures, obtained after 3 weeks of cell suspension. We looked at the percentage of CD133 and CD44 expression in each culture by fluorescence-activated cell sorting.

We did not detect CD133 expression on early and late cultures; conversely, CD44 was largely expressed (Table 2). We noticed an increase of CD44 expression during the 3 -week cell suspension; T early 'time 0 ' cultures presented a CD44 expression proportion of $56.12 \pm 34.47 \%$ SD (range: 25.7-97.8\%) which reached, in 3 weeks (late cultures), the $93.8 \pm 8.94 \%$ SD (range: 83.5-99.5\%) (Fig. 4). Moreover, we noticed that $\mathrm{N}+$ early cultures started at 'time 0 ' with a

Table 2. Characteristics of the six patients from whom cell cultures have been obtained and cultures' expression of CD44 and CD133

\begin{tabular}{|c|c|c|c|c|c|c|}
\hline Case & 1 & 2 & 3 & 4 & 5 & 6 \\
\hline Age/Sex & $63 / \mathrm{F}$ & $31 / \mathrm{M}$ & $65 / \mathrm{M}$ & $63 / \mathrm{M}$ & $68 / \mathrm{F}$ & $58 / \mathrm{M}$ \\
\hline Smoke (p/y) & 35 & 4 & 12 & 100 & 0 & 22 \\
\hline Alcohol & Yes & No & Yes & Yes & No & No \\
\hline Initial stage (AJCC 2005) & T1N1M0 & T2N0M0 & TxN1M0 & T4bN2bM0 & T2N2bM0 & T4aN1M0 \\
\hline Tumour site & $\begin{array}{l}\text { Tongue } \\
\text { margin }\end{array}$ & $\begin{array}{l}\text { Tongue } \\
\text { margin }\end{array}$ & $\begin{array}{l}\text { Lymph node } \\
\text { metastasis }\end{array}$ & $\begin{array}{l}\text { Retromolar } \\
\text { trigone }\end{array}$ & Larynx & Oral mucosa \\
\hline Comorbidities & No & No & Yes & No & No & No \\
\hline \multicolumn{7}{|c|}{ Immunohistochemical analysis } \\
\hline CD44 & + & + & + & + & + & - \\
\hline CD133 & - & - & - & - & - & - \\
\hline \multicolumn{7}{|l|}{ Cultures obtained from } \\
\hline $\mathrm{T}$ & Yes & Yes & No & Yes & No & Yes \\
\hline $\mathrm{N}$ & No & No & Yes & No & Yes & No \\
\hline \multicolumn{7}{|c|}{ Fluorescence-activated cell sorting analysis CD44 (\%) } \\
\hline Early culture & 25.7 & 30.1 & 98.4 & 71.1 & 98.2 & 97.8 \\
\hline Late culture & 99.6 & 83.5 & - & - & - & - \\
\hline
\end{tabular}




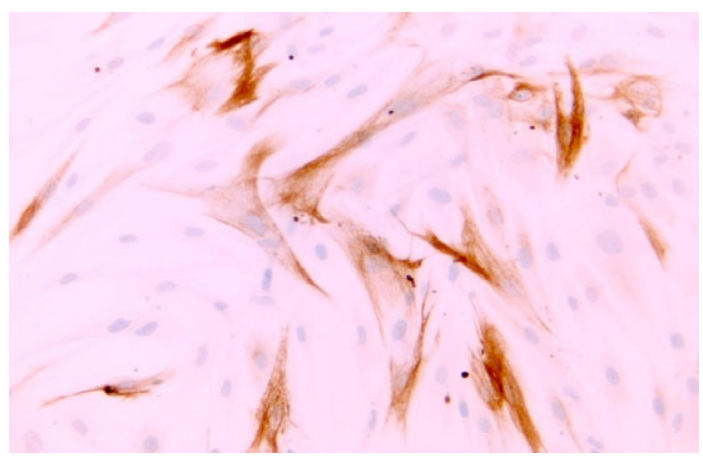

Fig. 3. Cell-specific cytokeratin-positive cancer cell cultures.

higher CD44 percentage of 98.33\% \pm 0.14 SD (range: $98.2-$ 98.4\%) $(P<0.05)$, highlighting a CD44 overexpression in early cultures from the lymph node metastasis (Fig. 4c).

\section{Discussion}

In this study, we evaluated the expression of CD44 and CD133 in biopsy specimens and early cell cultures from Head and neck squamous cell carcinoma by immunohis- tochemical assay and fluorescence-activated cell sorting analysis. We found a high percentage of CD44 expression (93.1\%) both in tumour specimens and in derived early cell cultures, confirming the diffuse distribution of this marker in Head and neck squamous cell carcinomas and a lower CD133 expression (10.34\%), similar to that reported in literature. ${ }^{9,10,32}$ Moreover, we established a successful rate of free-floating spherical colonies from primary tumours (21\%, four out 29) and from their corresponding cervical lymph node metastases $(\mathrm{N}+)$ (10.5\%, 2 out 19), higher than other Head and neck squamous cell carcinomas studies. ${ }^{33}$

In this setting, we documented the increasing expression of CD44+ subpopulations through several in vitro cultural steps, showing a clear clonogenic property (Fig. 4), that is their capacity to regenerate themselves through cell division. We also identified that cell clonogenicity was more evident in metastatic lymph node cell cultures than in those from primitive tumour, suggesting a possible more aggressive attitude of CD44-positive cancer stem cells obtained from $\mathrm{N}+$ specimens (Fig. 4c) and its potential involvement in metastatic process. (a)

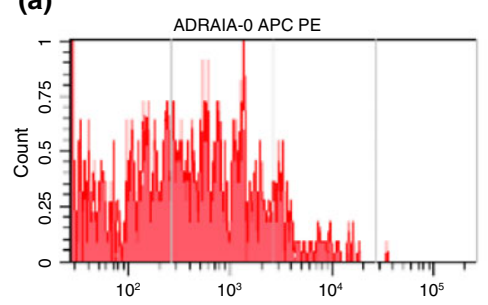

(b)

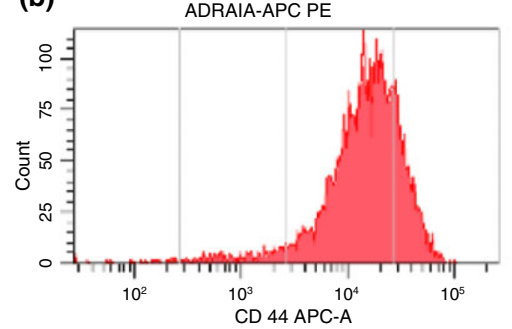

(c)

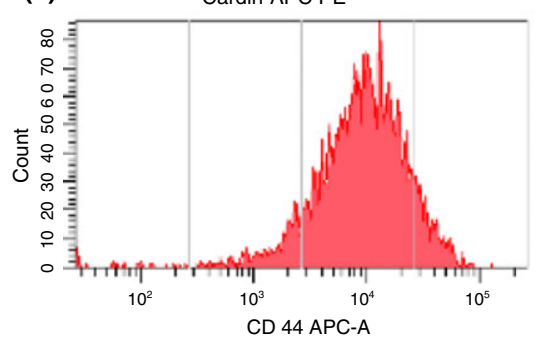

\begin{tabular}{|lrrr|}
\hline Tube: 0 APC PE & & & \\
Population & \#Events & \% Parent & $\%$ Total \\
All events & 20000 & $\# \# \# \#$ & 100.0 \\
$\square$ P1 & 1031 & 5.2 & 5.2 \\
\hline P2 & 826 & 80.1 & 4.1 \\
\hline P3 & 678 & 65.8 & 3.4 \\
P AND P3 & 674 & 65.4 & 3.4 \\
Q 11 & 2 & 0.3 & 0.0 \\
Q2 & 3 & 0.4 & 0.0 \\
Q2 & 496 & 73.6 & 2.5 \\
Q3 & 173 & 25.7 & 0.9 \\
\hline Q4 & & & \\
\hline
\end{tabular}
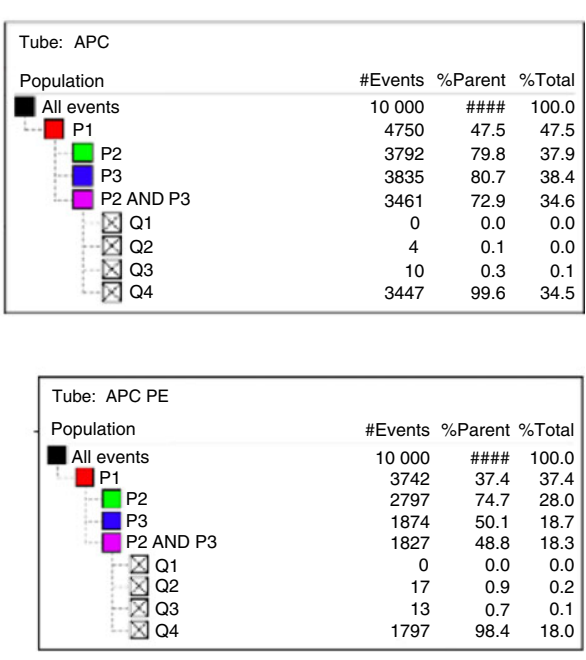

Fig. 4. Here, the most representative fluorescence-activated cell sorting analysis obtained for each different sample is reported. (a) T early culture with CD44 expression of 25.7\%; (b) T late culture with CD44 expression of 99.6\%; (c) CD44 expression in immunohistochemical early culture from lymph node metastasis. 
According to recent report, ${ }^{34}$ we demonstrated that Head and neck squamous cell carcinoma-driven squamouspheres exhibit some of cancer stem cell-like criteria, that is: (i) cancer stem cells-surface markers: CD44/CD133, (ii) squamouspheres-forming capacity, (iii) enrichment in CD44 expression at fluorescence-activated cell sorting analysis after in vitro culture passages and (iv) evidence of an existing starting subpopulation with near $98 \%$ expression of CD44 antigene in cervical lymph node metastases cultures.

Our findings regarding the high enrichment and the in vitro clonogenicity of $\mathrm{CD} 44+$ cells by $\mathrm{N}+$ specimens compared with results from primary tumour specimens are in agreement with increased motility in CD44-positive cancer cells in Head and neck squamous cell carcinoma, as recently reported by Davis SJ and Brown RL, ${ }^{35,36}$ highlighting a central role of CD44+ cancer stem cells in Head and neck squamous cell carcinomas progression and locoregional spreading. Accordingly, in a recent study on breast cancers, ${ }^{37}$ the standard isoform of CD44 antigen (CD44s) seems to be critical for regulating epithelial-mesenchymal transition, which is a process by which epithelial cells lose their polarity and are converted to a mesenchymal phenotype, and this process seems to be abnormally activated during cancer metastasis and recurrence. This process has been also regarded as the critical event to induce morphogenetic changes during embryonic development, organ fibrosis and tumour metastasis. ${ }^{38}$ In fact, the increased motility of metastatic cancer cells is typical of cells undergoing epithelial-mesenchymal transition. ${ }^{36}$ Taken together, these data suggest that regulation of CD44 expression could contribute to epithelial-mesenchymal transition and cancer progression, and it appears that cancer stem cells may have these capabilities; furthermore, our results might explain the enrichment of CD44+ cancer stem cells in lymph node metastases in in vitro study. ${ }^{37}$

Unfortunately, in this preliminary analysis, we did not identify CD133-positive cells in our limited number of cell cultures established. Few recent studies, ${ }^{10,33}$ mainly analysing in vitro Head and neck squamous cell carcinoma cell lines, have reported that CD44+/CD133+ cells are those with the best chance to represent the true cancer stem cell population also in Head and neck squamous cell carcinomas, as well as in other human solid tumours. ${ }^{39-43}$ The lack of fluorescence-activated cell sorting identification of this specific subpopulation in our cultures seems to reflect the low presence of this cancer stem cell marker in Head and neck squamous cell carcinoma initiating tumour cells as reported in literature. ${ }^{10,33}$ Another possible explanation of this lack is that it would be due to the coincidence that unfortunately all the harvested cell cultures were obtained from CD133-negative Head and neck squamous cell carcinomas, thus suggesting at least a less chance to detect or expand in vitro this specific and very rare subpopulation of Head and neck squamous cell carcinoma cells. Accordingly, others reported that CD133 marker is less expressed than CD44 in Head and neck squamous cell carcinomas, and its sensibility of identify cancer stem cells is still object of discussion. There are few studies in literature which have tried to identify cancer stem cells in Head and neck squamous cell carcinoma using CD133 surface marker, and they succeeded in $<10 \%$ of cases analysed. At variance, only CD44 as stem cell marker constitutively expressed on the surface of all permanent Head and neck squamous cell carcinoma cell lines, and thus, it may play a key role in establishment of permanent Head and neck squamous cell carcinoma cell lines. ${ }^{44}$ Anyway, some authors confirm CD133 as cancer stem cells marker. In fact, Harper et al. ${ }^{33}$ tested stem cell patterns in Head and neck squamous cell carcinoma cell lines through in vitro analysis by performing fluorescence-activated cell sorting analysis of potential stem cell markers (CD44, CD133 and CD29) in six Head and neck squamous cell carcinoma cell lines, and they obtained a subpopulation with high expression of CD44 for each cell line, while the CD133 expression was less intense. Yu et al. ${ }^{45}$ tried to identify the highly tumourigenic cell population in laryngeal carcinoma cells obtained from primary tumours using CD44/CD133 cell population. Wei et al. ${ }^{12}$ identified CD133-positive cancer cells with stem properties in laryngeal cancer cell lines (Hep-2); also, Okamoto et al. ${ }^{10}$ proposed CD133 antigen as a co-marker together with CD44 and ABCG2. Another recent paper in human oral squamous cancer analysed the cancer stem cells CD133-positive role in determining chemoresistance properties, further suggesting a possible key role of these cancer stem cells in Head and neck squamous cell carcinoma tumourigenesis. ${ }^{46}$ More recently, Chikamatsu et al. ${ }^{47}$ reported that CD44+ head and neck cancer stem cells were resistant to various apoptosis stimuli.

By our preliminary and limited results, we can add that tumour specimens showing the highest CD133 expression by immunohistochemical assay were those from cervical lymph node metastases $(10.5 \%, 2$ of $19 \mathrm{~N}+$ cases $)(P<0.05)$. This result, together with our immunohistochemical, cell cultures and fluorescence-activated cell sorting analyses, could help in identifying cell populations with morphologic stem celllike characteristics. Furthermore, this study suggests that CD44+ and CD133+ cells are detectable in Head and neck squamous cell carcinomas with a higher frequency in lymph node metastases than in primary tumours, thus suggesting a some role in Head and neck squamous cell carcinoma progression. This hypothesis of metastasising capacity and aggressive attitude of cancer stem cells, mainly of CD44+ cancer stem cell population, is further supported by our in vitro study, demonstrating that CD44+-cultured cells have the highest clonogenic capacity and are the main population 
proliferating in in vitro cultures obtained by $\mathrm{N}+$ Head and neck squamous cell carcinoma metastases.

Obviously, these preliminary results need to be confirmed in a larger series and need to be confirmed in experimental animal models.

\section{Keypoints}

- A putative cancer-stem-like-cell-population is detectable in HNSCCs.

- IHC and fluorescence-activated-cell-sorting-(FACS) analysis helped in counting CD44/CD133-CSCs markers percentage expression in HNSCC tumor-derived cultures and in identifying their clonogenic capacity.

- CD44+ cultured cells are the main population proliferating obtained by N+ HNSCC metastases, emphasizing their possible role in tumor progression.

\section{Acknowledgements}

This manuscript is approved by all authors, and all of them have participated in writing and correcting of this work. Further, all authors assure that manuscript has not been published nor is under approving by other journals or editors and they have not any conflict of interest, financial or otherwise.

\section{Conflicts of interest}

None to declare.

\section{References}

1 Parkin D.M., Bray F., Ferlay J. et al. (2005) Global cancer statistics, 2002. CA Cancer J. Clin. 55, 74-108

2 Ginos M.A., Page G.P., Michalavicz B.S. et al. (2004) Identification of a gene expression signature associated with recurrent disease in squamous cell carcinoma of the head and neck. Cancer Res. 64, 55-63

3 Gallo O., Chiarelli I., Bianchi S. et al. (1996) Loss of p53 gene mutation after irradiation is associated with increate aggressiveness in recurring head and neck cancer. Clin. Cancer Res. 2, 1577-1582

4 Gallo O., Boddi V., Calzolari A. et al. (1996) bcl-2 protein expression correlates with recurrence and survival in early stage head and neck cancer treated by radiotherapy. Clin. Cancer Res. 2, 261-267

5 Hamburger A.W. \& Salmon S.E. (1997) Primary bioassay of human tumor stem cells. Science 197, 461

6 Lapidot T., Sirard C., Vormoor J. et al. (1994) A cell initiating human acute myeloid leukaemia after transplantation into SCID mice. Nature 6464, 645-648

7 Morris R.J., Fisher S.M. \& Slaga T.J. (1986) Evidence that a slowly cycling subpopulation of adult murine epidermal cells retains carcinogen. Cancer Res. 46, 3061-3066
8 Clarke M.F. \& Fuller M. (2006) Stem cells and cancer: two faces of eve. Cell 124, 1111-1115

9 Prince M.E., Sivanandan R., Kaczorowski A. et al. (2007) Ailles LE Identification a subpopulation of cells with cancer stem cell properties in head and neck squamous cell carcinoma. PNAS 3, 973-978

10 Okamoto A., Chikamatsu K., Sakakura K. et al. (2009) Expansion and characterization of cancer stem-like cells in squamous cell carcinoma of the head and neck. Oral Oncol. 45, 633-639

11 Pries R., Wittkopf N., Trenkle T. et al. (2008) Potential stem cell marker CD44 is constitutively expressed in permanent cell lines of head and neck cancer. In Vivo 22, 89-92

12 Wei X.D., Zhou L., Cheng L. et al. (2009) In vivo investigation of CD133 as a putative marker of cancer stem cells in Hep-2 cell line. Head Neck 31, 94-101

13 Cotsarelis G., Kaur P., Dhouailly D. et al. (1999) Epithelial stem cells in the skin: definition, markers, localization and functions. Exp. Dermatol. 8, 80-88

14 Singh S.K., Hawkins C., Clarke I.D. et al. (2004) Identification of human brain tumour initiating cells. Nature 432, 396-401

15 Miki J., Furusato B., Li H. et al. (2007) Identification of putative stem cell markers, CD133 and CXCR4, in hTERT-immortalized primary non-malignant and malignant tumor-derived human prostate epithelial cell lines and in prostate cancer specimens. Cancer Res. 67, 3153-3156

16 O’Brien C.A., Pollett A., Gallinger S. et al. (2007) A human colon cancer cell capable of initiating tumour growth in immunodeficient mice. Nature 445, 106-110

17 Yin S., Li J., Hu C. et al. (2007) CD133 positive hepatocellular carcinoma cells possess high capacity for tumorigenicity. Int. J. Cancer 120, 1444-1450

18 Zhou L., Wei X., Cheng L. et al. (2007) CD133, one of the markers of cancer stem cells in Hep-2 cell line. Laryngoscope 117, 455-460

19 Shipitsin M., Campbell L.L., Argani P. et al. (2007) Molecular definition of breast tumor heterogeneity. Cancer Cell 11, 259-273

20 Pirinen R., Hirvikoski P., Böhm J. et al. (2000) Reduced expression of CD44v3 variant isoform is associated with unfavorable outcome in non-small cell lung carcinoma. Hum. Pathol. 31, 1088-1095

21 Kuhn S., Koch M., Nübel T. et al. (2007) A complex of EpCAM, claudin-7, CD44 variant isoforms, and tetraspanins promotes colorectal cancer progression. Mol. Cancer Res. 5, 553-567

22 Kalish E.D., Iida N., Moffat F.L. et al. (1999) A new CD44v3containing isoform is involved in tumor cell growth and migration during human breast carcinoma progression. Front Biosci. 4, A1-A8

23 Lucinei R.O., Jeffrey S.S. \& Ribeiro-Silva A. (2010) Review, Stem cells in human breast cancer. Histol. Histopathol. 25, 371-385

24 Prince M.E., Sivanandan R., Kaczorowski A. et al. (2007) Identification of a subpopulation of cells with cancer stem cell properties in head and neck squamous cell carcinoma. Proc. Natl Acad. Sci. USA 104, 973-978

25 Hill A., McFarlane S., Johnston P.G. et al. (2006) The emerging role of CD44 in regulating skeletal micrometastasis. Cancer Lett. 237, 1-9

26 Shmelkov S.V., Butler J.M., Hooper A.T. et al. (2008) CD133 expression is not restricted to stem cells, and both CD133+ and CD133- metastatic colon cancer cells initiate tumors. J. Clin. Invest. 118, 2111-2120

27 Abraham B.K., Fritz P., McClellan M. et al. (2005) Prevalence of CD44+/CD24-/low cells in breast cancer may not be associated with clinical outcome but may favor distant metastasis. Clin. Cancer Res. 11, 1154-1159 
28 Pardal R., Clarke M.F. \& Morrison S.J. (2003) Applying the principles of stem-cell biology to cancer. Nat. Rev. Cancer 3, 895-902

29 Locke M., Heywood M., Fawell S. et al. (2005) Retention of intrinsic stem cells hierarchies in carcinoma-derived cell line. Cancer Res. 65 8944-8950

30 Wang S.J., Wong G., de Heer A.M. et al. (2009) CD44 variant isoforms in head and neck squamous cell carcinoma progression. Laryngoscope 119, 1518-1530

31 Statistical Science, Inc. (1991) S-Plus Reference Manual. Statistical Science, Inc., Seattle, WA

32 Sterz C.M., Kulle C., Dakic B. et al. (2010) A basal-cell-like compartment in head and neck squamous cell carcinomas represents the invasive front of the tumor and is expressing MMP-9. Oral Oncol. 46, 116-122

33 Harper L.J., Piper K., Common J. et al. (2007) Stem cell patterns in cell lines derived from head and neck squamous cell carcinoma. J. Oral Pathol. Med. 36, 594-603

34 Young C.L., Se-Yeong O., Yun Y.C. et al. (2011) Cancer stem cells traits in squamouspheres derived from primary head and neck squamous cell carcinomas. Oral Oncol. 47, 83-91

35 Zhang S., Balch C., Chan M.W. et al. (2008) Identification and characterization of ovarian cancer-initiating cells from primary human tumors. Cancer Res. 68, 4311-4320

36 Davis S.J., Divi V., Owen H.H. et al. (2010) Metastatic potential of cancer stem cells in head and neck squamous cell carcinoma. Arch. Otolalryngol. Head Neck Surg. 136, 1260-1266

37 Brown R.L., Reinke L.M., Damerow M.S. et al. (2011) CD44 splice isoform switching in human and mouse epithelium is essential for epithelial-mesenchymal transition and breast cancer progression. J. Clin. Invest. 3, 1064-1074
38 Thiery J.P. \& Sleeman J.P. (2006) Complex networks orchestrate epithelial-mesenchymal transitions. Nat. Rev. Mol. Cell Biol. 7, 131-142

39 Zito G., Richiusa P., Bommarito A. et al. (2008) In vitro identification and characterization of CD133pos cancer stem-like cells in anaplastic thyroid carcinoma cell lines. PLoS ONE 3, 3455-3544

40 Fillmore C.M. \& Kuperwasser C. (2008) Human breast cancer cell lines contain stem-like cells that self-renew, give rise to phenotypically diverse progeny and survive chemotherapy. Breast Cancer Res. 10, $1-13$

41 Ponnusamy M.P. \& Batra S.K. (2008) Ovarian cancer: emerging concept on cancer stem cells. J. Ovarian Res. 1, 1-9

42 Lee C.J., Dosch J. \& Simeone D.M. (2008) Pancreatic Cancer Stem Cells. J. Clin. Oncol. 26, 2806-2812

43 Chan K.S., Espinosa I., Chao M. et al. (2009) Identification, molecular characterization, clinical prognosis, and therapeutic targeting of human bladder tumor-initiating cells. PNAS 106, 14016-14021

44 Prince R., Wittkopf N., Hasselbacher K. et al. (2008) Constitutive expression of the potential stem cell marker CD44 in permanent HNSCC cell lines. HNO 56, 461-466

45 Yu D., Jin C.S., Chen O. et al. (2009) Biological characteristics of highly tumorigenic CD44+CD133+ subpopulation of laryngeal carcinoma cells. Zhonghua Zhong Liu Za Zhi 31, 99-103

46 Zhang Q., Shi S., Yen Y. et al. (2010) A subpopulation of CD133(+) cancer stem-like cells characterized in human oral squamous cell carcinoma confer resistance to chemotherapy. Cancer Lett. 289, $151-160$

47 Chikamatsu K., Ishii H., Takahashi G. et al. (2012) Resistance to apoptosis-inducing stimuli in CD44+ head and neck squamous cell carcinoma cells. Head Neck 34, 336-343 